\title{
The gap between rod and frame influences the rod-and-frame effect with small and large inducing displays
}

\author{
PIERLUIGI ZOCCOLOTTI, GABRIELLA ANTONUCCI, and DONATELLA SPINELLI \\ Università "La Sapienza" Rome, Italy and Centro Ricerche, Clinica S. Lucia, Rome, Italy
}

\begin{abstract}
The role of the spatial separation between the ends of a rod and a frame's inner edge (gap) in modulating the rod-and-frame effect (RFE) has been studied here with frames subtending either large or small retinal angles. With a large frame, rod settings were always in the direction of frame tilt (direct effects) and varied inversely with gap size. With a small frame, rod settings were in the direction of frame tilt for tilts between $7.5^{\circ}$ and $15^{\circ}$; with larger frame tilts, rod settings in the direction opposite that of the frame were observed (indirect effects). Increasing gap size produced a tendency toward negativity (away from frame tilt). Consequently, direct effects were larger for small gaps, while the opposite was true for indirect effects. Overall, these results point to the importance of gap size in modulating the RFE, for both large and small displays.
\end{abstract}

In recent years, research on the rod-and-frame effect (RFE) has been directed by the hypothesis of a dualprocess model. According to this interpretation, in the case of inducing displays subtending large visual angles (approximately $20^{\circ}$ or more), RF phenomena would be mediated by visuovestibular interaction; in contrast, in the case of frames subtending small visual angles (approximately $10^{\circ}$ or less), visual mechanisms would be more prominent (for a review, see Spinelli, Antonucci, Goodenough, Pizzamiglio, \& Zoccolotti, 1991).

The role of visuovestibular interaction in mediating RF phenomena has been well documented (e.g., Bischof, 1974; Goodenough, Cox, Sigman, \& Strawderman, 1985). Consistent with this idea, it has been found that the standard RFE stimulus produces an illusion of selftilt in the direction opposite that of the frame (Ebenholtz \& Benzschawel, 1977; Sigman, Goodenough, \& Flannagan, 1978, 1979) and ocular torsion in the direction of the frame (Crone, 1975; Goodenough, Sigman, Oltman, Rosso, \& Mertz, 1979; Hughes, 1973). Both of these responses may be explained in terms of visuovestibular interactions.

The role and the nature of the visual mechanisms appear to be more controversial. One possibility is that the RFE is modulated by the general figural properties of the display. Streibel, Barnes, Julness, and Ebenholtz (1980) compared standard inducing displays (subtending about $30^{\circ}$ ) with a subjective contour condition. Equivalent form organization was not a sufficient condition for obtaining

This research was supported by grants from CNR and MPI. We would like to thank Don Goodenough for his critical reading of an earlier version of this paper and for his encouragement of this research project. We would also like to thank $M$. Basile and N. Crescenzi for their help in collecting data. Requests for reprints should be sent to P. Zoccolotti, Dipartimento di Psicologia, Via dei Marsi 78, 00185 Roma, Italy. equivalent effects on rod-adjustment errors. They concluded that "organizational or global factors that usually govern form perception are not effective determinants of orientation perception"' (p. 139). However, the role of the visual figural properties of the display was underscored by Johnstone and Wenderoth (1989), who used a $6^{\circ}$ "crossed-grating plaid," which produced angular function of the tilt illusion similar to that observed with the RFE. In this case, errors in rod settings were not determined by the direction of either of the two component gratings; rather, they depended upon the orientation of the virtual axes of the inducing figure, thus pointing to the presence of global effects in perception of the vertical.

Another possibility is that at least part of the RFE may be explained in terms of the relational properties between rod and frame. In particular, Ebenholtz (1985) referred to Koffka's (1935) idea of relational determination according to which "the apparent orientation of the line is largely predicated on the specific angle the line makes relative to a frame edge"' (p. 303). Ebenholtz reasoned that, if this theory was correct, the manipulation of the ease with which the angular relation of line to frame can be detected should produce direct effects on the size of the illusory effect. In two experiments, Ebenholtz used a $35.3^{\circ}$ frame and rods of decreasing size in order to produce a spatial separation between the ends of the line and the frame's inner edge (hereafter termed gap) of $14.3^{\circ}, 10.6^{\circ}$, and $3.1^{\circ}$. In both cases, inconsistent with the relational determination theory, the size of the RFE was independent of gap size. In a third experiment, using a larger frame $\left(54.6^{\circ}\right)$, Ebenholtz found that the effect was greater when a rod subtended a large visual angle $\left(49.7^{\circ}\right)$ than when a rod subtended a small visual angle $\left(6.0^{\circ}\right)$. This effect was present in the overall sample as well as in the subsample of high RFE scorers; for low scorers, the effect was small and not significant. However, probably in view 
of the negative results of the first two experiments, he did not interpret this finding as being due to the spatial separation between the frame and the rod; rather, he suggested that, impinging on the peripheral retina, the long rod contributed to modifying the subjective vertical of the observer. In other words, he interpreted the greater RFE in Experiment 3 in the long-rod (small gap) condition as being due to a visuovestibular, and not an intravisual effect.

In commenting upon Ebenholtz's (1985) study, Coren and Hoy (1986) noted that the absence of a gap effect had been obtained using spatial separations of more than $3^{\circ}$ of visual angle between the rod and the frame. On the contrary, orientation contrast effects are known to be maximal within the range of $1^{\circ}-2^{\circ}$ (Carpenter \& Blakemore, 1973). The presence of contrast orientation effects in the RFE illusion was clearly demonstrated by Goodenough, Oltman, Sigman, Rosso, and Mertz (1979), who used a visual-kinesthetic matching task. In that study, the observer aligned an invisible hand-held rod with a visual rod at various tilts under a control condition when the frame was absent, and under experimental conditions with the frame upright or set at $45^{\circ}$. The frame induced matching errors in the direction away from the frame sides which were most nearly parallel to the rod. ${ }^{1}$ The authors suggested that the observed RF illusion was similar to the angular expansion and contraction phenomena present in many traditional optical orientation illusions (e.g., the Zoellner illusion). It is relevant to the present discussion that a very small gap $\left(0.5^{\circ}\right.$ of visual angle) was used in this experiment.

In their study on the role of gap size, Coren and Hoy (1986) used a paper-and-pencil analog of the RFE. Even in the presence of ambient visual cues, small but reliable errors in adjusting a rod to the vertical were observed in the presence of a $6^{\circ}$ frame. In a second experiment, using a $6^{\circ}, 10^{\circ}$, or $15^{\circ}$ frame with a fixed $4^{\circ}$ long rod, Coren and Hoy found that with increasing frame size (and consequently increasing spatial rod-frame separation), the size of the illusory effect progressively decreased. This pattern of results appeared to be consistent with an interpretation in terms of a contrast orientation effect.

More generally, Coren and Hoy (1986) suggested that the results of their study and Ebenholtz's studies on the role of gap size support the dual-process model in indicating a predominance of visual mechanisms in central vision. However, in order to accept the conclusion that gap size is a factor that affects performance on small but not large RFE displays, it seems critical that the RFE be tested also with a relatively small gap in the large frame. However, apart from other differences (such as the use of classical or "paper-and-pencil" paradigms), the two studies differed along both critical parameters (size of the gap and size of the frame), preventing a firm conclusion.

Furthermore, Coren and Hoy's (1986) observation that a gap in the range of $1^{\circ}-2^{\circ}$ may be critical in producing orientation contrast effects suggests a tentative interpretation of the results of Ebenholtz's (1985) Experiment 3, alternative to what he proposed. In fact, in Ebenholtz's Experiment 3, the spatial separation between the rod ends and the frame was approximately $1.5^{\circ}$ in the case of the large rod and a significant difference between rods of different lengths was observed. ${ }^{2}$ This observation calls for a reconsideration of the importance of the gap factor in the case of large peripheral frames.

\section{EXPERIMENT 1}

The aim of the present study was to examine the role of gap size using both displays subtending a large and a small visual retinal angle. Following Coren and Hoy's (1986) suggestion, we selected gaps starting from $1^{\circ}$ to $2^{\circ}$ of visual angle for which the largest orientation contrast effects were expected.

\section{Method}

Subjects. Sixteen 20- to 30-year-old volunteers participated in the study.

Apparatus and Stimuli. Two RFE devices were built-one with 96.5-cm-long sides (large frame) and one with $9.7-\mathrm{cm}-$ long sides (small frame). Three rods were used $(92,81.6$, and $21 \mathrm{~cm}$ ) for the large frame and two rods were used $(7.6$ and $3.7 \mathrm{~cm})$ for the small frame. For the large frame, both the frame and the rod were made of a 1.2-cm-wide luminescent tape; for the small frame, the rod and frame were both $0.6 \mathrm{~cm}$ wide. In both cases, the rod and the frame were the only visible elements in an otherwise darkened room.

The frame and the rod were coplanar and could be independently pivoted around the center. The tilts of the frame and the rod could be read by the experimenter to the nearest half degree.

In the small-frame conditions, the observer was positioned $92 \mathrm{~cm}$ away from the stimulus; thus the frame sides subtended a visual angle of $6^{\circ}$. Gap sizes, defined here as the spatial separation between the end of the rod and the inner edge of the top (or bottom) of the frame, were measured with a frame orientation of $0^{\circ}$ and with the rod vertical, subtending $0.3^{\circ}$ and $1.5^{\circ}$ of visual angle. We refer to these two conditions as small and large gaps, respectively (see Figures 1 and 3 ). In the large-frame conditions, the observer was positioned $110 \mathrm{~cm}$ away from the apparatus; thus the frame sides subtended a visual angle of $47.4^{\circ}$. Gap sizes subtended $0.5^{\circ}$, $3.1^{\circ}$, and $18.3^{\circ}$ of visual angle (small, medium, and large, respectively, in Figure 1). ${ }^{3}$

Procedure. Each subject took part in two 1-h sessions. The subject was seated on a chair with his/her head supported in a vertical position by a headrest. Ten frame tilts were used $-37.5^{\circ}, 30^{\circ}$, $22.5^{\circ}, 15^{\circ}$, and $7.5^{\circ}$ clockwise; $7.5^{\circ}, 15^{\circ}, 22.5^{\circ}, 30^{\circ}$, and $37.5^{\circ}$ counterclockwise. In each case, the rod could initially appear with either a $37.5^{\circ}$ counterclockwise or a $37.5^{\circ}$ clockwise tilt. This produced a total of 20 different visual displays, which were repeated twice for each gap condition.

For the small-frame condition, a block of 20 trials with the small gap and one with the large gap was presented in each session. For the large-frame condition, 20 trial blocks of small, medium, and large gaps were presented in each session. This produced a total of 80 trials in the small-frame condition and 120 trials in the largeframe condition. A brief rest period was allowed between blocks. For both frame conditions, block sequences were counterbalanced across subjects.

For all conditions, the order of presentation of the stimuli (frame tilt $\times$ rod position) was randomized separately within each block for each subject. The subjects were instructed to direct the experimenter in aligning the rod with the gravitational vertical. The rod was manually moved by small steps in either direction, as indicated 
by the subject. Bracketing was permitted; no time limit was given. Errors in rod adjustment to the vertical were used to measure performance. Preliminary analyses of variance (ANOVAs) indicated nonsignificant differences between clockwise and counterclockwise frame orientations. Therefore, for the purpose of analysis, errors were considered as positive if they were in the direction of frame tilt, and negative if they were opposite the frame tilt.

\section{Results}

Rod settings for all conditions are shown in Figure 1. These results confirm a number of previous findings. In particular, the number of errors in rod settings in the largeframe condition was greater than that for the small-frame condition. Consistent with previous studies, in the case of the large frame, the observers always set the subjective vertical in the direction of the frame tilt. Peak effect was at $15^{\circ}$ of frame tilt - a finding consistent with previous results of within-subject studies examining the shape of the function relating rod-setting errors to frame tilts (Goodenough et al., 1985; Zoccolotti, Antonucci, Goodenough, Pizzamiglio, \& Spinelli, 1992). In the case of the small frame, errors in rod settings were in the direction of frame orientation for small-frame tilts $\left(7.5^{\circ}, 15^{\circ}\right)$ and in the opposite direction for larger tilts-a pattern of results consistent with Wenderoth's $(1974,1977)$ data for similar frame size.

To evaluate the role of gap size, mean algebraic errors in rod settings were submitted to two separate ANOVAs for the two frame conditions. In both cases, the analysis considered frame orientation $\left(7.5^{\circ}, 15^{\circ}, 22.5^{\circ}, 30^{\circ}\right.$, and $37.5^{\circ}$ ) and gap size as repeated factors (for the large-frame condition, three levels of gap were considered; for the small-frame, two levels).

Large frame. The main effect of gap size was significant $[F(2,30)=10.55, p<.001]$. The large gap produced a smaller effect $\left(1.4^{\circ}\right)$ than the medium $\left(1.7^{\circ}\right)$ and small one $\left(2.0^{\circ}\right)$ did (in both cases, $p<.01$, Duncan test); these latter values did not differ from each other. The main effect of frame orientation was also significant $[F(4,60)=16.28, p<.0001]$. The interaction between the two factors was not significant $[F(8,120)=1.75, p=$ .09] (see Figure 1).

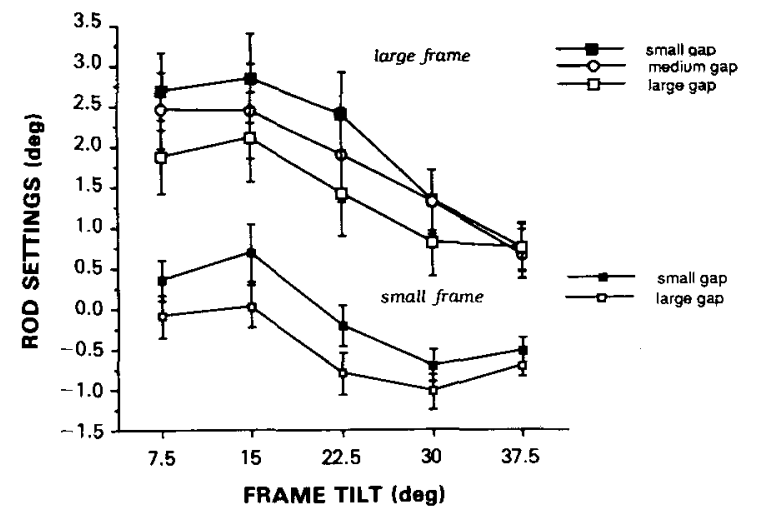

Figure 1. Mean rod settings as a function of frame tilt for different gap sizes for both the large and small frames. Positive values indicate errors in the direction of frame tilt, negative values indicate the opposite; bars indicate standard errors.
Small frame. The main effect of gap size was significant $[F(1,15)=10.8, p<.01]$; mean rod settings were $-.08^{\circ}$ for the $.3^{\circ}$ gap and $-.52^{\circ}$ for the $1.5^{\circ}$ gap. The main effect of frame orientation was significant $[F(4,60)=$ $10.4, p<.0001]$. The interaction between the two factors was not significant $(F<1)$.

Using a standard RFE apparatus, it can be observed that gap size was not equal across frame tilts and with rod orientation. That is, absolute gap sizes increased with increasing frame tilt. Rod-setting errors for both large- and small-frame conditions are plotted in Figure 2 as a function of the actual gap size corresponding to each frame tilt (see Note 3). An inspection of the figure clearly indicates that, for both large- and small-frame conditions, rodsetting errors tended to decrease with increasing gap size. This decrement is greater for gap sizes of less than $3^{\circ}-4^{\circ}$ and is less for larger gap sizes.

\section{Discussion}

The main result of the present study is that the gap between the inner edge of the frame and the rod ends is a factor modulating the RFE for inducing frames of both large and small eccentricity. In the case of a large peripheral frame, these findings are inconsistent with those reported in Ebenholtz's (1985) Experiments 1 and 2 (but not in Experiment 3). Following Coren and Hoy's (1986) suggestion, it seems likely that the use of a gap of less than $2^{\circ}$ of visual angle for the large-frame condition may have been critical in detecting the role of gap size. In fact, it is within this range of spatial separation that most clearcut orientation contrast effects have been reported (Carpenter \& Blakemore, 1973). However, it is noteworthy that small but reliable orientation effects have also been reported for spatial separations of up to $5^{\circ}-6^{\circ}$ (Hartley, 1982; Wenderoth, Johnstone, \& van der Zwan, 1989). These findings may help in understanding the progressive reduction of rod-setting errors in all three gap conditions for the large-frame display. ${ }^{4}$

An alternative interpretation accounting for gap-size effects has been proposed by Ebenholtz (1985). In commenting on the results of his third experiment, he suggested that in the small-gap condition the longer rods, impinging on the peripheral retina, might contribute to the magnitude of the RFE by enhancing visuovestibular interactions. Ebenholtz noted (1985, Note 4) that the gap effect was clearer in high RFE scorers. This may explain part of our data, but it does not easily account for the overall pattern observed. In fact, gap size is effective with the short rod of the small-frame condition and also in the range of frame tilts for which indirect effects were present. Moreover, if one assumes that the final rod position is the one contributing most of the effect, ${ }^{5}$ rod tilts averaging approximately $2^{\circ}-3^{\circ}$ (such as the ones found in the large-frame condition) should contribute very little in terms of visuovestibular interactions. In fact, these diminish linearly with decreasing frame tilt (Goodenough et al., 1985), suggesting that tilts of the rod in a $2^{\circ}-3^{\circ}$ range are expected to produce minimal visuovestibular effects. 


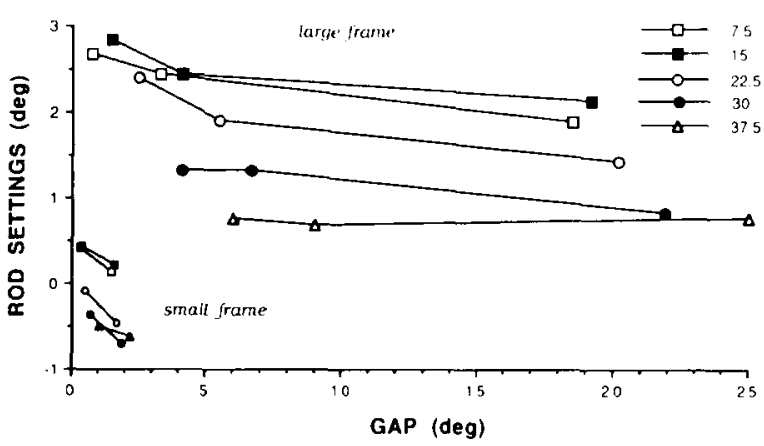

Figure 2. Mean rod settings as a function of gap size for different frame tilts and for both the large and small frames (for details on the calculation of gap size, see Note 3). Positive values indicate errors in the direction of frame tilt, negative values indicate the opposite.

As for the small central frame, the present findings generally confirm those of Coren and Hoy (1986). However, a number of differences among the two studies must also be noted. In Coren and Hoy's study, observers viewed a paper-and-pencil analog of the RF stimulus in full daylight. Since the illuminated room provided strong gravitational reference axes, it is unlikely that visuovestibular mechanisms were activated by the tilted frame in this condition; consequently, visual effects were presumably the sole determinant of the illusory effect. This observation may account for the unusual finding that the illusory effect progressively vanished with increasing frame size (with a parallel increase in the gap between rod and frame). In agreement with what has commonly been found for standard presentation in the dark (e.g., Ebenholtz \& Benzschawel, 1977), we also observed a large increase in the illusory effect with increasing frame size.

A further difference between our study and Coren and Hoy's (1986) regards the shape of the function of rod setting and frame orientation. In the case of a small frame subtending approximately $6^{\circ}$ of visual angle, Wenderoth (1974) reported that rod settings erred in the direction of frames between $0^{\circ}$ and $22^{\circ}$ (direct effects) and in the opposite direction between $22^{\circ}$ and $45^{\circ}$ of frame tilt (indirect effects). The precise conditions of occurrence and the nature of indirect effects are still unclear. In general, they have been reported with very small frames subtending $6^{\circ}$ of visual angle or less; with frames of approximately $10^{\circ}$, direct effects are observed most often (e.g., Zoccolotti et al., 1992).

On the basis of the ambiguity of the frame as a visual stimulus, Wenderoth (1974) proposed that observers may see a $35^{\circ}$ clockwise tilt frame as a diamond with a counterclockwise tilt. Consequently, between $22^{\circ}$ and $45^{\circ}$ of frame tilt, observers may base their judgments on the (perceived) diagonal of the diamond. Wenderoth (1977) showed that the presence of the corners is critical for producing indirect effects. More generally, within the dualprocess model, it has been proposed that configurational factors, which are supposed to be irrelevant in the case of the large peripheral frame, may exert a role in the case of small centrally fixated displays (Coren \& Hoy, 1986; Ebenholtz \& Glaser, 1982; Wenderoth et al., 1989). In Coren and Hoy's study, rod settings were always in the direction of frame tilts; in contrast, in the present research, a pattern closely resembling that described by Wenderoth (1974) was observed. No clear interpretation of this different outcome is readily apparent; frame size was approximately the same in the two studies. Furthermore, indirect effects have never been interpreted in terms of visuovestibular interaction; consequently, an interpretation based on the difference between RF stimuli presented in either a dark or an illuminated room also seems unlikely.

The nature of the interpretation of indirect effects is directly relevant to understanding the role of gap in the present experiment. Unlike what we found for the smaller gap, with the larger gap, the whole curve describing rodsetting errors as a function of frame tilt was "pushed down" toward negativity (i.e., away from frame orientation). Because of this, in frame tilts of $7.5^{\circ}$ and $15^{\circ}$, illusory effects in the direction of frame tilt were greater in the smaller gap than in the larger gap. With larger frame tilts, rod settings erred in the direction opposite that of the frame. These indirect effects were more pronounced in the large gap than in the small gap.

Considering the variability in obtaining indirect effects, we made no explicit prediction as to the role of gap size for frame tilts above $22^{\circ}$. One a posteriori interpretation is that a longer rod, more closely approaching the perceived "top side" of the frame, is more effective in countering the perceptual shift of perceiving the frame as a counterrotated diamond. With larger gaps, rods may appear less linked to the side of the frame and an alignment to the frame corners (i.e., diagonal of the "diamond") may be more important. Relevant to this interpretation is the observation by Wenderoth (1974) that, in the case of frames of approximately $6^{\circ}$ of visual angle, frame "corners" may be a more critical factor in orientation perception than the alignment of the sides of the frame which are least displaced from the gravitational vertical (as in the case of visuovestibular interaction). He consistently found that the typical curve of the RFE for the small frame could be closely reproduced by truncations of the frame, which would include two adjacent sides of the frame (phenomenologically appearing as a corner or an arrow); in contrast, truncations maintaining two parallel sides of the frame produced a qualitatively different curve of rod-setting errors as a function of frame tilt.

\section{EXPERIMENT 2}

The pattern of results in the case of the small frame was unpredicted, so we decided to replicate the finding in a second study.

\section{Method}

Subjects. Twenty 20- to 30-year-old volunteers participated in the study.

Stimuli and Procedure. These were exactly the same as those used in Experiment 1. 


\section{Results and Discussion}

Rod settings for all conditions are shown in Figure 3. The main effect of gap size was significant $[F(1,19)=$ $8.3, p<.011$; mean rod settings were $-.3^{\circ}$ for the $.3^{\circ}$ gap and $-.02^{\circ}$ for the $1.5^{\circ}$ gap. The main effect of frame orientation was significant $[F(4,76)=12.71, p<.0001]$. The interaction between the two factors was not significant $(F<1)$.

The results of Experiment 2 indicate a difference between the two gap conditions that is virtually identical to that of Experiment 1. Overall, these findings point to the reliability of the observed influence of the gap factor on small RFE stimuli.

\section{GENERAL DISCUSSION}

From a general theoretical standpoint, the present results appear to be inconsistent with at least some versions of the dual-process model that have been proposed to account for the RFE. According to some authors (Coren \& Hoy, 1986; Ebenholtz \& Glaser, 1982), visuovestibular interactions should be present only in large peripheral frames; in contrast, visual interactions should be restricted to small central RF displays. Although a precise match between the size of the gaps in the large- and small-frame conditions could not be accomplished, there were no indications in the present data that the effect was confined to the case of the frame subtending a smaller visual angle. However, it should be observed that the present results are not inconsistent with a less stringent version of the model, proposed by Goodenough et al. (1979). According to these authors, visuovestibular interactions are at work only in the case of large displays, but intravisual effects are present independent of frame size.

It may be added that we recently gathered further evidence that also appears to be inconsistent with the visuovestibular part of the dual-process model (Zoccolotti et al., 1992). We presented large and small RF stimuli to observers when they were upright or lying on their sides; with horizontal observers, the size of the RFE increased dramatically, confirming previous research (Good-

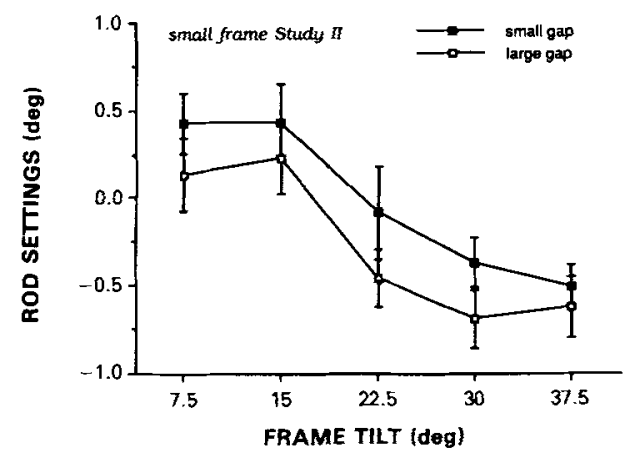

Figure 3. Mean rod settings as a function of frame tilt for different gap sizes for a small frame. Positive values indicate errors in the direction of frame tilt, negative values indicate the opposite; bars indicate standard errors. enough et al., 1985). Because otolith sensitivity varies inversely with body tilt, this increased illusory effect is interpreted as being due to visuovestibular interaction (Bischof, 1974; Goodenough et al., 1985). Inconsistent with the dual-process model, exactly the same increase in effect was present in the case of a large peripheral frame and of a small central frame. Jointly, the results of these two studies show that, even though it may be easier to detect the role of visuovestibular and intravisual mechanisms in large and small frames, respectively, both kinds of influence can be proven on frames of very different sizes, provided that the appropriate experimental conditions are met. In the case of gap, it has been observed that large gaps were used in most experiments using the large-frame condition (Spinelli et al., 1991); conversely, researchers using small frames typically used small gaps (e.g., Wenderoth, 1977).

This observation is important for interpreting previous studies that appeared to provide support for the dualprocess model. For example, working with a small RFE apparatus, Gogel and Newton (1975) found a depth adjacency effect-errors in aligning a rod to the vertical were more pronounced when the rod and the frame were coplanar than when they were located at different distances from the observer. This depth adjacency effect was confirmed in the case of small but not large frames by Ebenholtz and Glaser (1982). However, independent of depth differences, small frames in this experiment had considerably smaller gaps than larger frames did. Therefore, considering the influence of gap on the size of the RFE and its independence from frame size, the results of the present experiment suggest that qualitative differences among inducing frames subtending different retinal angles should be tested, controlling for this factor.

\section{REFERENCES}

Bischof, N. (1974). Optic-vestibular orientation to the vertical. In H. H. Kornhuber (Ed.), Handbook of sensory physiology: Vestibular system: Pt. 2. Psychophysics, applied aspects and general interpretations (pp. 155-190). New York: Springer-Verlag.

Carpenter, R. H. S., \& Blakemore, C. (1973). Interactions between orientation in human vision. Brain Research, 18, 287-303.

COREN, S., \& HoY, V. S. (1986). An orientation illusion analog to the rod and frame: Relational effects in the magnitude of the distortion. Perception \& Psychophysics, 39, 159-163.

Crone, R. A. (1975). Optically induced eye torsion: II. Optostatic and optokinetic cycloversion. Albrecht von Graefes Archive für Klinische und Experimentelle Ophthalmologie, 196, 1-7.

EbEnholtz, S. M. (1985). Absence of relational determination in the rod-and-frame effect. Perception \& Psychophysics, 37, 303-306.

Ebenholtz, S. M., \& Benzschawel, T. L. (1977). The rod and frame effect and induced head tilt as a function of observation distance. Perception \& Psychophysics, 22, 491-496.

Ebenholtz, S. M., \& Glaser, G. W. (1982). Absence of depth processing in the large-frame rod-and-frame effect. Perception \& Psychophysics, 32, 134-140.

Gogel, W. C., \& Newton, R. E. (1975). Depth adjacency and the rod-and-frame illusion. Perception \& Psychophysics, 18, 163-171.

Goodenough, D. R., Cox, P. W., Sigman, E., \& Strawderman, W. E. (1985). A cognitive-style conception of the field-dependence dimension. Cahiers de Psychologie Cognitive, 5, 687-706. 
Goodenough, D. R., Oltman, P. K., Sigman, E., Rosso, J., \& MERTZ, H. (1979). Orientation contrast effects in the rod-and-frame test. Perception \& Psychophysics, 25, 419-424.

Goodenough, D. R., Sigman, E., Oltman, P. K., Rosso, J., \& MERTZ, H. (1979). Eye torsion in response to a tilted visual stimulus. Vision Research, 19, 1177-1179.

HARTLEY, A. A. (1982). The roles of axes of symmetry in orientation illusions. Perception \& Psychophysics, 31, 367-375.

Hughes, P. C. (1973). The influence of the visual field upon the visual vertical in relation to ocular torsion of the eye. (Doctoral dissertation, University of Oklahoma, 1974). Dissertation Abstracts International, $33,4686 \mathrm{~B}$

Johnstone, S., \& WENDERoth, P. (1989). Spatial and orientation specific integration in the tilt illusion. Perception, 18, 5-23.

KoFfKA, K. (1935). Principles of gestalt psychology. New York: Harcourt, Brace

Sigman, E., Goodenough, D. R., \& Flannagan, M. (1978). Subjective estimates of body tilt and rod-and-frame test. Perceptual \& Motor Skills, 47, 1051-1056.

Sigman, E., Goodenough, D. R., \& FlannaGan, M. (1979). Instructions, illusory self-tilt and the rod-and-frame test. Quarterly Journal of Experimental Psychology, 31, 155-165.

Sinelu, D., Antonucci, G., Goonenough, D. R., Pizzamiglio, L., \& Zoccolotti, P. (1991). Psychophysiological mechanisms underlying the rod and frame illusion. In S. Wapner \& J. Demick (Eds.), Field dependence-independence: Cognitive style across the life span (pp. 37-60). Hillsdale, NJ: Erlbaum.

Streibel, M. J., Barnes, R. D., Julness, G. D., \& Ebenholtz, S. M. (1980). Determinants of the rod-and-frame effect: Role of organization and subjective contour. Perception \& Psychophysics, 27, 136-140.

WeNDEROTH, P. (1974). The distinction between the rod-and-frame illusion and the rod-and-frame test. Perception, 3, 205-212.

WENDEROTH, P. (1977). An analysis of the rod-and-frame illusion and its variants. In R. H. Day \& G. V. Stanley (Eds.), Studies in perception (pp. 95-141). Perth: University of Western Australia Press.

WENDEROTh, P., JohNSTONE, S., \& VAN DER ZWAN, R. (1989). Twodimensional tilt illusion induced by orthogonal plaid patterns: Effects of plaid motion, orientation, spatial separation, and spatial frequency. Perception, 18, 25-38.
Zoccolotti, P., Antonucci, G., Goodenough, D., Pizzamiglio, L., \& SPinelli, D. (1992). The role of frame size on upright \& tilted observers in the rod and frame test. Acta Psychologica, 79, 171-187.

\section{NOTES}

1. Consequently, in order to appear vertical, the rod should be moved in the direction of frame tilt; in other words, even if the nature of the mechanism is different, at least within a range of frame tilts, contrast orientation and visuovestibular mechanisms are expected to produce errors in verticality judgments in the same direction.

2. Ebenholtz (1985) did not explicitly report the size of the gap for this experiment. The frame side measured $106.5 \mathrm{~cm}$, and the two rods measured 95.5 and $10.8 \mathrm{~cm}$. Considering that line thickness of the frame was $2.4 \mathrm{~cm}$, spatial separation between the inner edge of the frame was either 3.1 or $45.4 \mathrm{~cm}$, which, at a distance of $103.2 \mathrm{~cm}$. corresponds to $1.7^{\circ}$ and $25.2^{\circ}$ of visual angle, respectively.

3 . The gap varies with frame and rod tilt according to the following formula: $g=\left[\left(L_{4}-2 S_{f}\right) / 2 \cos (a-c)\right]-L_{\mathrm{f}} / 2$, where $g$ is the distance measured along the virtual prolungation of the rod between the end of the rod and the inner edge of the frame and represents the gap size; $L_{\mathrm{f}}$ and $L_{\mathrm{r}}$ represent the length of frame and rod, respectively; $S_{\mathrm{f}}$ is the thickness of the frame; and $a$ and $c$ represent the frame and rod tilt with respect to the vertical. If the final setting of the rod is the one that is important to gap size, we might assume that the $c$ value contributes little to the gap size and simplify the above expression to $g=\cdot\left[\left(L_{\mathrm{f}}-2 S_{\mathrm{f}}\right) / 2 \cos a\right]-$ $L_{\tau} / 2$. In Figure 2, the RFE is presented as a function of gap size for the various conditions of frame tilts using this formula.

4. Further work using the visual-kinesthetic matching technique developed by Goodenough et al. (1979) to detect contrast orientation effects independent of visuovestibular interaction may prove particularly helpful in confirming the presence of visual orientation effects for RF displays in the case of gaps of up to $5^{\circ}-6^{\circ}$

5. Initial rod position cannot easily account for the effects found, because an equal number of clockwise and counterclockwise rod initial tilt positions were used.

(Manuscript received December 16, 1991; revision accepted for publication December 4,1992 .) 